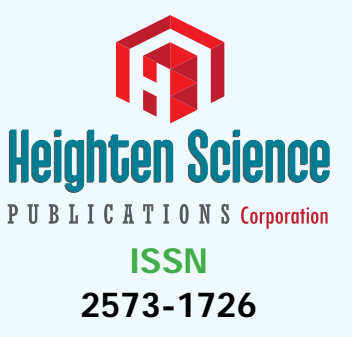

*Address for Correspondence: Guilherme M Cesar, Movement and Neurosciences Center at the Institute for Rehabilitation Science and Engineering, Madonna Rehabilitation Hospital. 5401 South St, Lincoln, NE, 68506-2150, United States, Tel: (402) 413-4503; Email: gcesar@madonna.org

Submitted: 25 February 2017

Approved: 18 March 2017

Published: 21 March 2017

Copyright: @ 2017 Cesar GM, et al. This is an open access article distributed under the Creative Commons Attribution License, which permits unrestricted use, distribution, and reproduction in any medium, provided the original work is properly cited.

Keywords: Injury risk assessment; Vertical jump; Motion analysis; Anterior cruciate ligament

\title{
3-Dimensional Versus 2-Dimensional Comparison of Knee Valgus Collapse during Vertical J ump: Clinical Implications for ACL Risk of Injury
}

\section{Assessment}

\author{
Guilherme M. Cesar ${ }^{1 *}$, Chase M. Pfeifer ${ }^{1,2}$ and J udith M. \\ Burnfield ${ }^{1}$ \\ Institute for Rehabilitation Science and Engineering, Madonna Rehabilitation Hospital, 5401 \\ South St, Lincoln, NE, 68506-2150, USA \\ 2Department of Mechanical and Materials Engineering, University of Nebraska-Lincoln, W342 \\ NH, Lincoln, NE, 68588-0526, USA
}

\section{ABSTRACT}

Time-efficient screening of lower extremity biomechanics to identify potential injurious movement patterns is crucial within athletic medicine settings. When considering biomechanical risk factors for anterior cruciate ligament injuries, several screening tests have been used to assess dynamic knee valgus. Current methods involving 3-dimensional motion capture systems are considered gold standard for such assessment; however, these methods are time consuming and require expensive materials. This study investigated the use of 2-dimentional kinematic evaluation during a standardized vertical jump athletic assessment to screen for potential lower extremity risk of injury. 50 collegiate athletes, 25 male and 25 female, from various sports participated in the study. The vertical jump was chosen because it is a common performance evaluation test that is regularly performed several times a year, providing consistent opportunities for screening while not creating additional obligations for the student athletes. Results showed that the 2-dimentional evaluation method had strong correlations $(P \varangle 0.0001$ ) with the gold standard 3-dimensional evaluation, suggesting that an accelerated 2-dimentional screening process can be used as a first step to screen for potential injurious lower extremity movement patterns.

\section{INTRODUCTION}

Time-efficient screening of lower extremity (LE) biomechanics to detect potential injurious movement patterns is crucial within athletic medicine settings. When considering biomechanical risk factors for anterior cruciate ligament (ACL) injuries, several screening tests have been used to assess dynamic knee valgus. Current methods involving 3-dimensional (3D) motion capture systems are considered gold standard for such assessment; however, these methods are time consuming and require expensive materials. Since observational assessments have been reported to be moderately reliable [1] and sensitive [2], 2-dimentional (2D) techniques have been used to evaluate LE biomechanics and objectively quantify risk factors of ACL injuries.

The amount of 2D motion of the knee joint in the frontal plane during dynamic tasks has been assessed with different populations, including young [3,4] and collegiate [5] athletes. Sigward and colleagues [6] investigated the association between 2D knee 
separation distance (KSD) and the average between right and left (i.e., bilateral average) knee valgus angles at peak knee flexion during the drop landing task and found that half of the variance observed in knee frontal plane angle could be explained by the 2D data after controlling for the participants' stance width. This finding further supports the use of 2D technique to facilitate time-efficient lower extremity assessments to promote health and safety for athletes at risk for ACL injuries.

A number of double-limb and single-limb landing tasks are generally used in the literature, along with side-step cutting to different angles [7], for the assessment of dynamic knee valgus, including the drop jump (e.g., [8], drop landing (e.g., [9], and single-leg landing (e.g., [10]). Recently, Cesar and colleagues [11] have shown that the vertical jump task exhibits similar lower extremity mechanics regarding the knee valgus collapse than the drop jump task. In fact, knee joint valgus angle and internal adductor moment observed during vertical jump were strongly correlated with the angle and moment observed during the drop jump task ( $\mathrm{r}=0.93$ and $\mathrm{r}=0.82$, respectively). Since vertical jump is a task consistently used across sport settings to assess athletic performance [12-14], utilizing such jumping maneuver concurrently for the assessment of potential risks of lower extremity injuries should enhance preventive interventions. Thus, verifying the sensitivity of the 2D assessment during vertical jump for the screening of dynamic knee valgus is warranted for its use in both clinical and sports performance settings.

While most studies utilize individuals with demonstrable valgus (e.g., [15]), it is not clear how sensitive the screening of 2D knee frontal plane motion would be with individuals exhibiting a more neutral alignment of the lower extremity. Moreover, a varus knee joint position has been detected in male athletes during different jumplanding tasks [16], which is a position also known to place injurious stress to the ACL fibers $[17,18]$. Therefore, the objective of our study was to detect the strength of the relationship between the 3D and 2D frontal plane knee motion during the vertical jump task of an athletic cohort including male and female participants exhibiting a large range of frontal plane motion (i.e., valgus and varus). Knee frontal plane excursions are usually larger in one joint compared with the other side [19]. Since most studies investigating the 2D KSD utilize the average angle obtained from both knees (e.g., [6]), we also investigated the relationship between the KSD and each knee joint. It was hypothesized that 3D bilateral average and unilateral frontal plane knee joint data would be negatively correlated with 2D KSD in the vertical jump task.

\section{METHODS}

\section{Subjects}

Ethical guidelines were followed and ethical approval for the study was granted by the University of Nebraska-Lincoln's Institutional Review Board (number 14863). Fifty ( 25 females) National Collegiate Athletic Association (NCAA) Division I studentathletes (basketball, football, volleyball, gymnastics) participated in this crosssectional study. Average ( \pm standard deviation) age, height, and mass were $19.33 \pm 1.33$ years, $183.58 \pm 13.52 \mathrm{~cm}$, and $88.11 \pm 21.74 \mathrm{~kg}$, respectively. Participants were healthy at the time of data collection with no history of lower extremity or trunk injuries in the previous 6 months. Exclusion criteria involved previous injury that resulted in ligamentous laxity at the hip, knee, or ankle joints, and presence of any medical or neurologic condition (e.g., concussion) that would impair the current ability to perform athletic maneuvers.

\section{Procedures}

Upon arrival study procedures were explained to each student-athlete and informed consent and parental assent were obtained before participation. Prior to testing, participants warmed up for approximately 7 minutes with drills involving jogging and 
dynamic stretching to full range of motion under the supervision of the team's strength and conditioning coach.

Reflective kinematic markers were then placed on each student-athlete. Twentythree markers were placed over anatomical landmarks of the participants' pelvis and lower extremity segments: bilaterally over the distal end of second and fifth metatarsal bones (on the shoe), heel (on the shoe), medial and lateral malleoli, tibial tuberosity, medial and lateral epicondyles of femur, a marker placed above the patella (one third of the distance between patella and anterior superior iliac spine), greater trochanters, iliac crests, and one marker over L5-S1 junction. Marker set-up can be seen in figure 1 . An eleven-camera motion analysis system (Qualisys $®$, Gothenburg, Sweden) was used to capture the 3D and 2D kinematic data sampled at $250 \mathrm{~Hz}$.

For the experimental task, participants performed the vertical jump as described previously [11]. Briefly, they were instructed to stand with their dominant hand closest to the Vertec device (Power Systems, Knoxville, TN), feet placed shoulderwidth apart, and arms raised overhead. After holding this posture for one second, participants were instructed to perform a single counter-movement and jump straight vertically to reach maximal height by targeting the highest possible vane on the Vertec with their dominant hand. The depth of the counter-movement was not controlled and each participant performed the counter-movement to their comfort. This task was performed 3 times.

\section{Data analysis}

Kinematic data were processed in Visual3D ${ }^{\mathrm{TM}}$ (C-Motion, Inc., Rockville, MD, USA) and custom Matlab (Math Works, Natick, MA, USA) codes were used to identify variables used in this study. Marker trajectory data were filtered using a fourth order Butterworth low-pass filter with a $10 \mathrm{~Hz}$ cut-off frequency.

For the kinematic 3D data, local coordinate systems for the body segments (pelvis, thighs, shanks, and feet) were derived from the standing calibration trial taken prior to the vertical jump. Six degrees-of-freedom of each segment was determined from the segment's kinematic triad by transforming the triad of markers to the position and orientation of each segment determined from the standing calibration trial. Joint kinematics (angles) were calculated using a joint coordinate approach [20].

Four markers were used to calculate the 2D kinematic data: lateral epicondyle of femur and greater trochanter, bilaterally. KSD was calculated (in centimeters) as distance (in centimeters) in the frontal plane between the markers attached to right and left lateral epicondyles of the femur. To account for the effects of body type, KSD was normalized to the distance (in centimeters, Equation 1) in the frontal plane between the markers attached to right and left greater trochanters (intertrochanteric distance). The marker configuration used for the calculation of the normalized KSD (nKSD) is provided in figure 1 .

$$
n K S D=\frac{\text { Knee Separation Distance }}{\text { Intertrochanteric Distance }}
$$

Both 3D and 2D data considered for statistical analysis were derived from the software Qualisys and Visual3D, and collected at peak knee flexion angle. 3D data consisted of the average of both right and left knee joint frontal plane angles (i.e., bilateral average), and individual frontal plane angles for the right and left knee joints. The following convention was used to report knee frontal plane data at peak knee flexion: positive (+) valgus angle and negative (-) varus angle. All three trials were considered per participant.

\section{Statistical analysis}

Six trials ( 3 males and 2 females) were not considered for statistical treatment as the marker placed on the lateral epicondyle of the femur was occluded. A total of 144 trials 
were used and descriptive statistics at the time of peak knee flexion were calculated and expressed as mean, standard deviation, minimum and maximum values. Pearson correlation coefficient ( $\mathrm{r}$ ) was calculated to examine the relationship between 3D and 2D knee frontal plane motion at the time of peak knee flexion. Observed power (OP, $1-\beta$ ) was calculated to verify the strength of the inferences regarding the statistical treatments. A linear regression analysis was performed on the 3D knee joint frontal plane bilateral average to examine the association of the combined joint angle on the 2D nKSD. When both variables were evaluated separately (right and left 3D knee joint frontal plane angle), multiple linear regression was performed to examine the impact of each variable on nKSD. Statistical analyses were performed with IBM SPSS Version 22 statistical package, with significant levels set a priori at $\alpha=0.05$.

\section{RESULTS}

Detailed demographics of our participants can be seen in table 1.

Descriptive statistics for 2D nKSD and 3D knee joint frontal plane angles are provided in table 2 .

Significant negative correlation $(\mathrm{r}=-0.655 ; \mathrm{P}<0.001 ; \mathrm{OP}>0.99)$ was detected between $n K S D$ and 3D frontal plane angle bilateral average when using the Pearson correlation (Figure 2). Significant negative association was observed when performing a linear regression $\left(r=0.654 ; \mathrm{P}<0.001 ; \mathrm{F}_{1,142}=106.4\right)$. The following is the resulting prediction equation:

$$
3 D_{\text {angle }}=20.8-(16.0 * n K S D)
$$

In addition, significant negative correlations $(\mathrm{P}<0.001)$ were detected between $\mathrm{nKSD}$ and 3D frontal plane angle of the left $(\mathrm{r}=-0.650 ; 0 \mathrm{P}>0.99$; Figure $3 \mathrm{~A})$ and right $(\mathrm{r}=-$

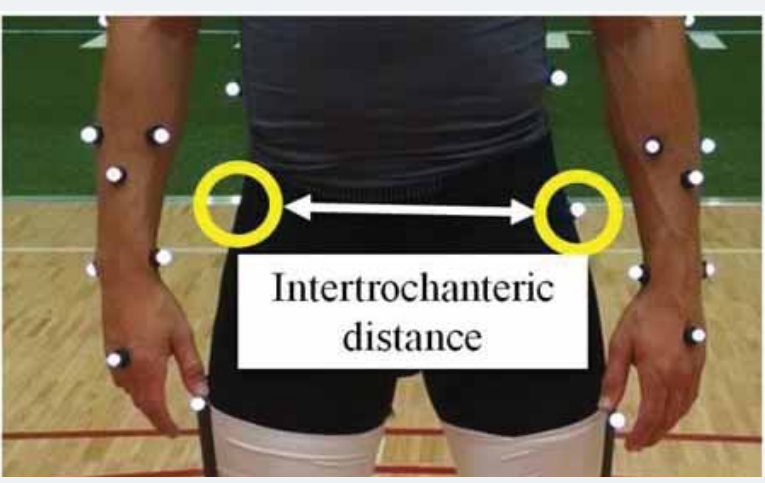

Figure 1: Three-dimensional (3D) kinematic marker setup. Markers used for the 2D calculation (intertrochanteric distance and KSD) are circled.

\begin{tabular}{|c|c|c|c|c|c|}
\hline Sport & Sample (sex) & Age (yrs) & Mass (kg) & Height (m) & BMI $\left(\mathrm{kg} / \mathrm{m}^{2}\right)$ \\
\hline Basketball & $11(\mathrm{~m})$ & 19.6 & 93.2 & 1.94 & 24.7 \\
\hline Football & $14(\mathrm{~m})$ & 20.4 & 109.2 & 1.89 & 30.0 \\
\hline Volleyball & $12(f)$ & 20.2 & 83.0 & 1.84 & 24.6 \\
\hline Soccer & $5(f)$ & 19.2 & 75.5 & 1.81 & 23.1 \\
\hline Gymnastics & $8(f)$ & 19.8 & 60.7 & 1.62 & 23.3 \\
\hline
\end{tabular}

\begin{tabular}{|l|l|l|l|l|}
\hline Table 2: Descriptive statistics for the variables of interest. (+) valgus angle and (- ) varus angle. \\
\hline & Mean & Std. Deviation & Minimum & Maximum \\
\hline Normalized Knee Separation Distance & 1.04 & 0.18 & 0.65 & 1.47 \\
\hline Average Frontal Plane Angle $\left(^{\circ}\right)$ & 4.12 & 4.46 & -8.66 & 11.58 \\
\hline Left Knee Frontal Plane Angle ( $\left.{ }^{\circ}\right)$ & 4.27 & 5.40 & -9.34 & 18.28 \\
\hline Right Knee Frontal Plane Angle $\left(^{\circ}\right)$ & 3.98 & 4.68 & -9.08 & 13.44 \\
\hline
\end{tabular}


0.497; OP>0.99; Figure 3B) knee joints when using the Pearson correlation. Significant negative association was observed when performing a multiple linear regression, most heavily predicted by the left knee $\left(r=0.669\right.$; Left $P<0.001$; Right $\left.P=0.011 ; F_{2,141}=57.1\right)$. The following is the resulting prediction equation:

$$
n K S D=1.15-\left(0.0182 * \text { Left } 3 D_{\text {angle }}\right)-\left(0.0076 * \text { Right } 3 D_{\text {angle }}\right)
$$

\section{DISCUSSION}

Efforts to enhance preventive measures against ACL injuries have increased in the past decades as this injury is highly detrimental to the individual on and off the court/ field. An effective screening mechanism is warranted to maintain athletes' health and well-being by preventing potential injuries to the knee joint. The findings of our study support that $2 \mathrm{D}$ assessment of movement in the frontal plane, while performing a vertical jump, could be used as a substitute for the 3D assessment when this technique is not available. Combining the simpler 2D screening (compared to the more expensive and time consuming 3D method) with a standard athletic performance evaluation, such as the vertical jump, could provide a valuable approach in screening for athletes that are at a higher risk of lower extremity injury.

In agreement with our hypothesis, we found a strong relationship [21] between 3D bilateral average and 2D (nKSD) knee joint motion in the frontal plane during the vertical jump task. The negative correlation between nKSD and the 3D variables indicates that lower $\mathrm{nKSD}$ values were demonstrative of valgus angles while a larger nKSD indicated a more neutral or varus angles. These results corroborate previous studies supporting the use of 2D assessment in athletic environments as relationship of 2D data measures with valgus angles were reported optimistically [6,5,22]. Our study contributes to this body of literature by providing a full range of frontal plane motion (i.e., valgus and varus angles) during a routinely performed jumping task.

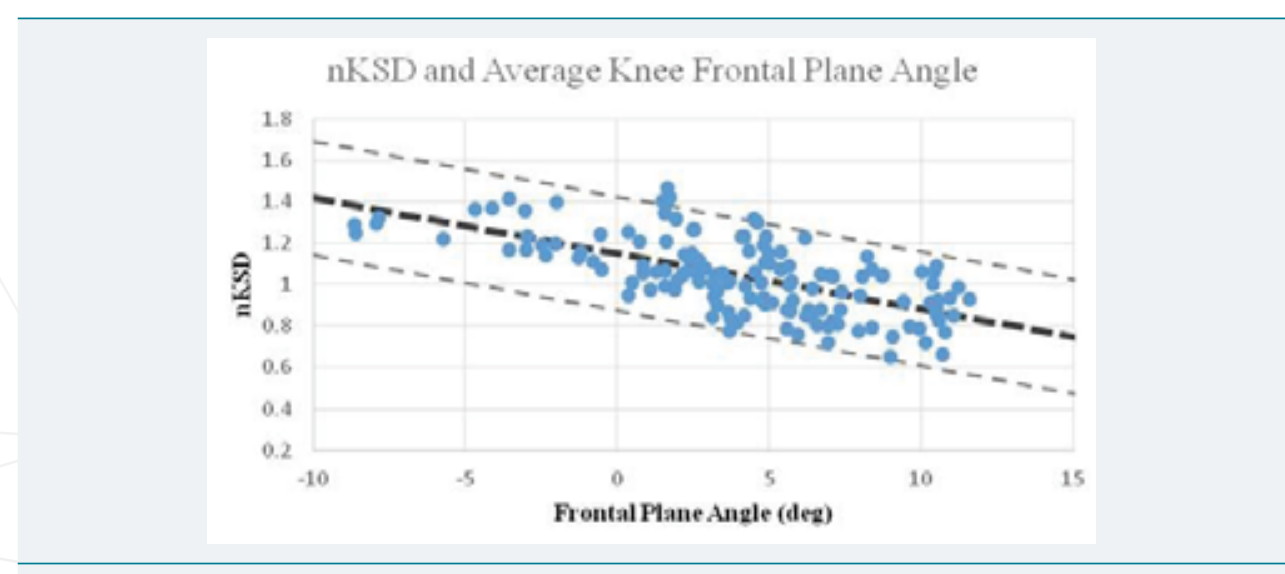

Figure 2: Relationship between three-dimensional (3D) bilateral average of knee joint frontal plane angle and knee separation distance normalized by intertrochanteric distance (nKSD).
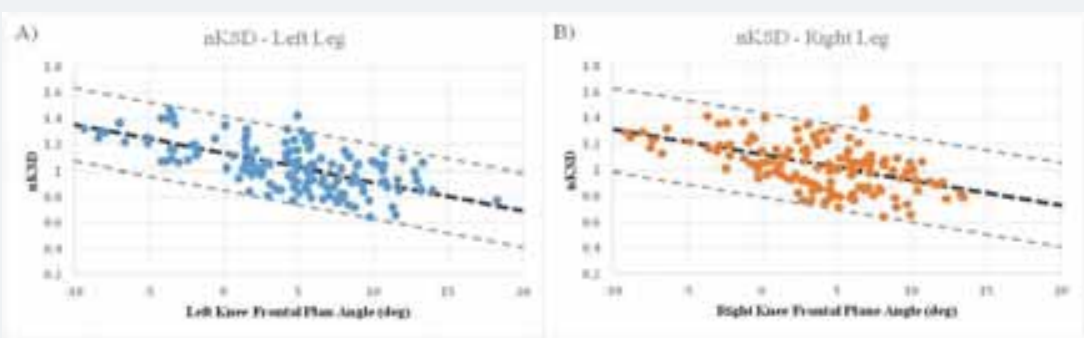

Figure 3: Relationship between knee separation distance normalized by intertrochanteric distance (nKSD) and 3D left $(A)$ and right $(B)$ knee joint frontal plane angle. 
Our study is the first to evaluate the 2D analysis of the vertical jump task with the purpose of screening for potential lower extremity injurious movement patterns. The vertical jump is a maneuver regularly used in current protocols to evaluate athletic performance [12]. It is done several times during pre-season and within the athletes' season to assess individual responses to training regimens. The use of this task simultaneously for the evaluation of ACL risk of injury would broaden athletes' safety while constantly testing their performance capabilities. Since the 2D analysis was strongly correlated with the $3 \mathrm{D}$ assessment, it is suggested that the $2 \mathrm{D}$ analysis of the vertical jump could be a feasible alternative for clinicians and athletic medicine staff to intensify preventive care when 3D assessments cannot be conducted. Moreover, when considering the athlete contact time restrictions delimited in the United States by the National Collegiate Athletic Association, the use of the 2D nKSD evaluation during regular performance testing periods, can provide a time-efficient assessment for a large number of athletes that otherwise may have not been screened for a risk of lower extremity injury.

Certain limitations to our study should be considered when interpreting our findings. First, participants were asked to maintain their feet aligned with their hip before performing the vertical jump. However, in order to not interfere with the athletes' performance, we were not able to control the motion of their feet (i.e., repositioning) during their counter-movement. This could influence the relationship between the markers used for 2D calculations at peak knee flexion, resulting in $n K S D \geq 1$. Future studies should determine whether incorporating stance width into the nKSD calculation [6] could provide more robust correlations during the vertical jump task. Second, although the precise contribution remains unknown, knee flexion angle can contribute to the resultant 2D frontal plane knee angle measure when the femur is internally rotated. Thus, larger valgus estimates from frontal plane projections are expected when the femur is in such a position. This observation suggests that 2D measurements should be considered with care when precise descriptions of knee valgus magnitudes are necessary. Lastly, we measured 2D knee separation distance from coordinate measures of reflective markers using high-speed motion capture cameras. This technique has been used in previous research studies to determine differences in knee valgus motion during drop landings. However, clinical screening of knee separation distance typically relies on still frame photos taken from standard video camera recordings. Therefore, the association between $2 \mathrm{D}$ knee separation distance measured clinically and actual 3D lower extremity angles may result in less accurate measures of knee separation distance than those reported in the current study.

In conclusion, this study provided an innovative approach towards preventive measures in a cohort of collegiate athletes. The $2 \mathrm{D}$ screening process can be used as a first step to assess potential injurious lower extremity movement patterns during a jumping task that is ubiquitously performed across different age groups and athletic environments. The vertical jump task is also regularly performed several times a year, providing consistent opportunities for coaches and trainers to monitor the progression of intervention protocols. Future work should investigate the development of mobile applications to quickly detect nKSD thresholds during vertical jump to provide the athletic medicine staff with valuable information to maintain athletes' safety and wellbeing across training regimens.

\section{REFERENCES}

1. Chmielewski TL, Hodges MJ, Horodyski M, Bishop MD, Conrad BP, et al. Investigation of clinician agreement in evaluating movement quality during unilateral lower extremity functional tasks: a comparison of 2 rating methods. J Orthop Sports Phys Ther. 2007; 37: 122-129. Ref.: https://goo.gl/ZQcuQ5

2. Ekegren $\mathrm{CL}$, Miller WC, Celebrini RG, Eng J J , Macintyre DL. Reliability and validity of observational risk screening in evaluating dynamic knee valgus. J Orthop Sports Phys Ther. 2009; 39: 665-674. Ref.: https://goo.gl/MWVea5 
3. Noyes FR, Barber-Westin SD, Fleckenstein C, Walsh C, West J. The drop-jump screening test: difference in lower limb control by gender and effect of neuromuscular training in female athletes. Am J Sports Med. 2005; 33: 197-207. Ref.: https://goo.gl/ADHyC7

4. Barber-Westin SD, Noyes FR, Galloway M. J ump-land characteristics and muscle strength development in young athletes: a gender comparison of 1140 athletes 9 to 17 years of age. Am J Sports Med. 2006; 34: 375-384. Ref.: https://goo.gl/WbSn9Y

5. MCLean SG, Walker K, Ford KR, Myer GD, Hewett TE, et al. Evaluation of a two dimensional analysis method as a screening and evaluation tool for anterior cruciate ligament injury. Br J Sports Med. 2005; 39: 355-362. Ref.: https://goo.gl/Tzuzpz

6. Sigward SM, Havens $\mathrm{KL}$, Powers $\mathrm{CM}$. Knee separation distance and lower extremity kinematics during a drop land: implications for clinical screening. J Athl Train. 2011; 46: 471-475. Ref.: https://goo.gl/aFS7TZ

7. Sigward SM, Cesar GM, Havens KL. Predictors of Frontal Plane Knee Moments during Side-Step Cutting to 45 and 110 Degrees in Men and Women: Implications for Anterior Cruciate Ligament Injury. Clin J Sport Med. 2015; 25: 529-534. Ref.: https://goo.gl/YoYW4C

8. Stearns $\mathrm{KM}$, Powers $\mathrm{CM}$. Improvements in hip muscle performance result in increased use of the hip extensors and abductors during a landing task. Am J Sports Med. 2014; 42: 602-609. Ref.: https://goo.gl/UoGzgB

9. Pollard CD, Sigward SM, Powers CM. Limited hip and knee flexion during landing is associated with increased frontal plane knee motion and moments. Clin Biomech (Bristol, Avon). 2010; 25: 142-146. Ref.: https://goo.gl/aM3HUU

10. Cesar GM, Pereira VS, Santiago PR, Benze BG, da Costa PH, et al. Variations in dynamic knee valgus and gluteus medius onset timing in non-athletic females related to hormonal changes during the menstrual cycle. Knee. 2011; 18: 224-230. Ref.: https://goo.gl/s7seq6

11. Cesar GM , Tomasevicz CL, Burnfield J M. Frontal plane comparison between drop jump and vertical jump: implications for the assessment of ACL risk of injury. Sports Biomech. 2016; 15: 440-449. Ref.: https://goo.gl/Cf8ARI

12. Nesser TW, WL Lee. The relationship between core strength and performance in division I female soccer players. J ournal of Exercise Physiology Online. 2009; 12: 21-28. Ref.: https://goo.gl/1sDxha

13. Robbins DW, Goodale TL, Kuzmits FE, Adams AJ. Changes in the athletic profile of elite college American football players.J Strength Cond Res. 2013; 27: 861-874. Ref.: https://goo.gl/Nazo4K

14. Keller RA, Mehran N, Austin W, Marshall NE, Bastin K, et al. Athletic Performance at the NFL Scouting Combine After Anterior Cruciate Ligament Reconstruction. Am J Sports Med. 2015; 43: 3022-3026. Ref.: https://goo.gl/NAITnh

15. Bell DR, Oates DC, Clark MA, Padua DA. Two- and 3-dimensional knee valgus are reduced after an exercise intervention in young adults with demonstrable valgus during squatting. J Athl Train. 2013; 48: 442-449. Ref.: https://goo.gl/UmlJ Dq

16. Chappell J D, Yu B, Kirkendall DT, Garrett WE. A comparison of knee kinetics between male and female recreational athletes in stop-jump tasks. Am J Sports Med. 2002; 30: 261-267. Ref.: https://goo.gl/8qt2um

17. Bendjaballah MZ, Shirazi-Adl A, Zukor DJ . Finite element analysis of human knee joint in varusvalgus. Clin Biomech. 1997; 12: 139-148. Ref.: https://goo.gl/R5Z6Ws

18. Markolf KL, Burchfield DM, Shapiro MM, Shepard MF, Finerman GA, et al. Combined knee loading states that generate high anterior cruciate ligament forces. J Orthop Res. 1995; 13: 930-935. Ref. https://goo.gl/dXAEwU

19. Ford KR, GD Myer, TE Hewett. Valgus knee motion during landing in high school female and male basketball players. Med Sci Sports Exerc. 2003; 35: 1745-1750. Ref.: https://goo.gl/WYqg5Y

20. Grood ES, WJ Suntay. A joint coordinate system for the clinical description of three-dimensional motions: Application to the knee. J ournal of Biomechanical Engineering. 1983; 105: 136- 144. Ref.: https://goo.gl/f7Pelf

21. Evans J D. Straightforward statistics for the behavioral sciences. Pacific Grove, CA, Brooks/Cole Publishing.1996; Ref.: https://goo.gl/a6j39l

22. Mizner RL, Chmielewski TL, Toepke JJ, Tofte KB. Comparison of 2-dimensional measurement techniques for predicting knee angle and moment during a drop vertical jump. Clin J Sport Med. 2012; 22: 221-227. Ref.: https://goo.gl/9sOsJ h 